\title{
Substituent effects on the through-space nuclear magnetic spin-spin coupling in van der Waals dimers
}

\author{
Alessandro Bagno, Giacomo Saielli, and Gianfranco Scorrano* \\ Department of Chemistry and Centro Meccanismi Reazioni Organiche del CNR, Via Marzolo, 1 \\ 35131, Padova, Italy \\ E-mail: gianfranco.scorrano@unipd.it
}

This paper is dedicated to Professor Dionis Sunko on the occasion of his $\mathbf{8 0}^{\text {th }}$ birthday

(received 04 Apr 02; accepted 22 Jul 02; published on the web 30 Jul 02)

\begin{abstract}
We have investigated the effect of substituents on the donor (benzene) and acceptor (C-H) moieties of $\mathrm{CH} / \pi$ interacting model systems 1-6 on the through-space nuclear spin-spin coupling $J_{\mathrm{CH}}$ and $J_{\mathrm{HH}}$. These couplings were recently predicted by ab-initio and DFT calculations to be of the order of $0.2-0.3 \mathrm{~Hz}$ at the equilibrium separation. Electronic effects due to the substitution are found not to increase significantly, and often to decrease, the calculated coupling, since they involve mainly $\pi$ orbitals, while the coupling is mainly transmitted through $\sigma$ orbitals.
\end{abstract}

Keywords: Through-space coupling, scalar coupling, $\mathrm{CH} / \pi$ interaction, DFT calculations

\section{Introduction}

Recently [1,2] we have investigated several model van der Waals system dimers showing the socalled $\mathrm{CH} / \pi$ interaction, where a $\mathrm{C}-\mathrm{H}$ bond is directed, more or less perpendicularly, towards a $\pi$ system, typically an aromatic ring. The model systems were comprised of a benzene ring or an ethylene molecule, as the electron density donors in the $\mathrm{CH} / \pi$ interaction, while the $\mathrm{CH}$ bond acceptors were represented by methane, ethylene, and benzene, respectively. For the smaller dimers, we ran a series of calculations at the ab-initio (RASSCF, Restricted Active Space Self Consistent Field and SOPPA, Second Order Polarization Propagator Approach) and DFT (Density Functional Theory) levels of theory. Nuclear spin-spin $J_{\mathrm{CH}}$ coupling was calculated between the hydrogen of the acceptor $\mathrm{CH}$ bond and the carbon atoms of the donor $\pi$ system, which belong to two different molecules. The calculations consistently predicted a through-space $J_{\mathrm{CH}}$ spin-spin coupling in the $\mathrm{CH} / \pi$ interacting dimers on the order of $0.2-0.3 \mathrm{~Hz}$, which is well above the limit of resolution of modern NMR spectrometers [3]. These results prompted us to extend the study to compounds known to have stable, long-lived, $\mathrm{CH} / \pi$ interactions, unlike the 
small model dimers, so that the NMR splittings may be detected within the lifetime of the compound.

The results of DFT calculations on some compounds stabilized by $\mathrm{CH} / \pi$ interaction indicate a relatively short through-space distance between a hydrogen and a carbon atom that otherwise are separated by a large number of bonds, in agreement with the corresponding results obtained for the model system dimers. They confirm that, also in these cases, the $J_{\mathrm{CH}}$ spin coupling must occur through space.

The cases we have studied, however, only involved unsubstituted moieties. In this paper, we present preliminary results concerning the effects of substituents, both on the aromatic donor part as well as on the acceptor $\mathrm{CH}$ bond of the $\mathrm{CH} / \pi$ interacting system.

\section{Results}

For this study we have selected the model dimers shown in Figure 1. All the monomers were optimized at the HF/6-31G* level of theory by using the Gaussian 98 software package [4]. The distance $R$ between the carbon of the $\mathrm{CH}$ bond and the center of the aromatic ring was set to 3.80 $\AA$, which is in the minimum potential well for interaction of the corresponding unsubstituted complexes [1,2].
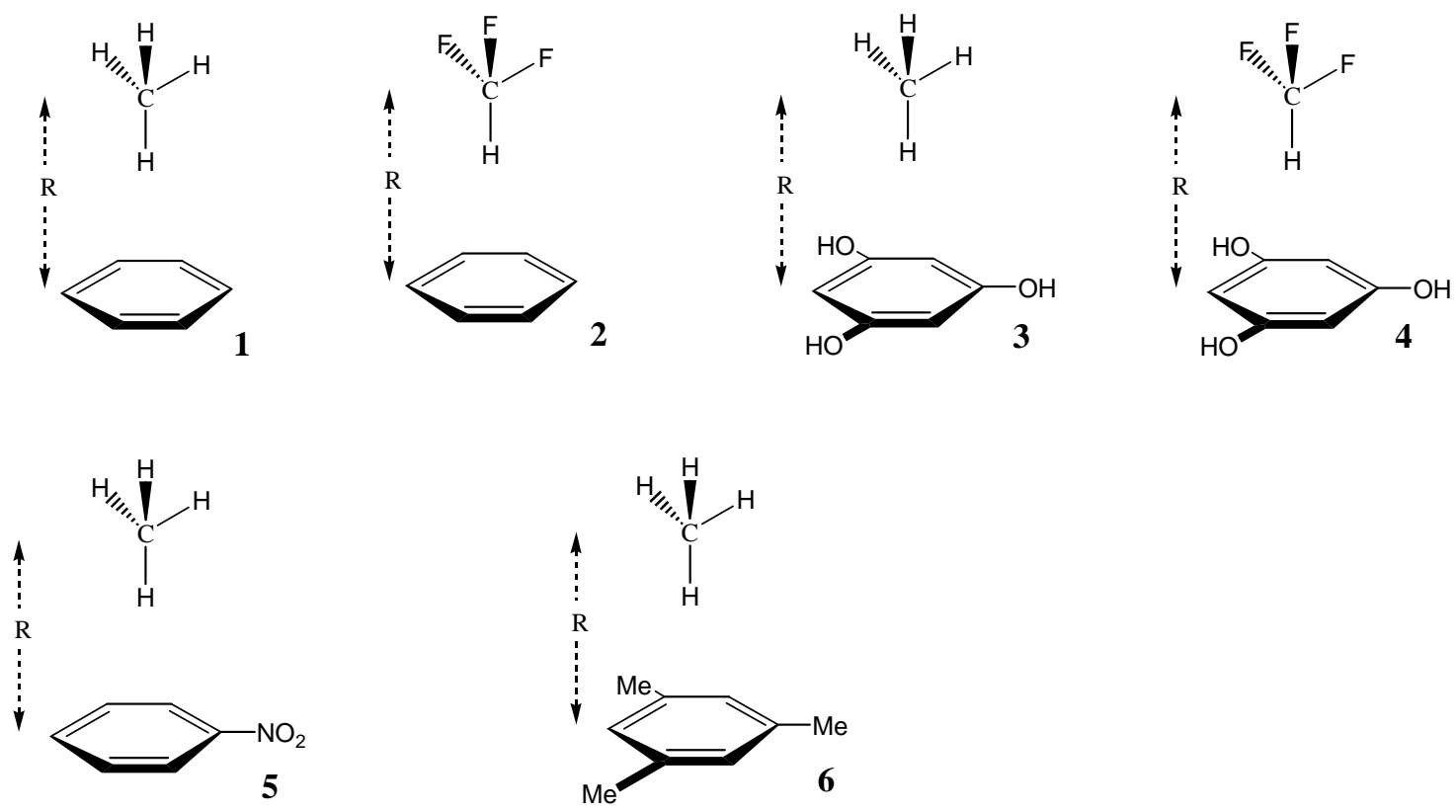

Figure 1. Schematic representation of the model dimers studied in this work.

It is useful, at this point, to recall some qualitative considerations on the effect of substituents on the energetics of the $\mathrm{CH} / \pi$ dimers [5]. If we consider the $\mathrm{CH} / \pi$ interacti on as a donor/acceptor interaction with a partial charge transfer from the HOMO of the donor (aromatic 
ring) to the LUMO of the acceptor ( $\mathrm{CH}$ bond), then substituents on the donor which increase the energy of the HOMO (electron-donating substituents) and/or substituents on the acceptor which decrease the energy of the LUMO (electron-withdrawing substituents) will increase the stability of the dimer. This is because more electron density is "pushed" onto the aromatic ring and/or more electron density is also "pulled" onto the $\mathrm{CH}$ bond. Therefore we expect the dimer $\mathbf{4}$ to be the most stable and the unsubstituted dimer $\mathbf{1}$ the least stable. However, it is by no means clear how these substituents can affect the through-space coupling. Therefore we have calculated $J_{\mathrm{CH}}$, as well as $J_{\mathrm{HH}}$, between the hydrogen of the $\mathrm{CH}$ bond and the carbon and hydrogen atoms of the aromatic ring. We have used the deMon-NMR software package [6] to calculate the spin-spin couplings at the VWN/IGLO-III level of theory, as in our previous studies [1,2]. The deMonNMR code allows for the calculations of the main contributions to the spin-spin couplings, namely the Fermi contact term (FC) and the diamagnetic (DSO) and paramagnetic (PSO) spinorbit contributions. The results of such calculations are reported in Table 1.

Table 1. Through-space spin-spin couplings $(\mathrm{Hz})$ for the dimers investigated

\begin{tabular}{|c|c|c|c|c|c|}
\hline & & FC & PSO & DSO & Total \\
\hline \multirow[b]{2}{*}{1} & $J_{\mathrm{CH}}$ & 0.09 & -0.28 & 0.38 & 0.19 \\
\hline & $J_{\mathrm{HH}}$ & 0.00 & -0.15 & 0.15 & 0.00 \\
\hline \multirow[b]{2}{*}{2} & $J_{\mathrm{CH}}$ & 0.05 & -0.38 & 0.53 & 0.21 \\
\hline & $J_{\mathrm{HH}}$ & 0.00 & -0.42 & 0.57 & 0.16 \\
\hline \multirow{3}{*}{3} & $J_{\mathrm{CH}}{ }^{(\mathrm{a})}$ & 0.04 & -0.45 & 0.52 & 0.11 \\
\hline & $J_{\mathrm{CH}}{ }^{(b)}$ & 0.11 & -0.39 & 0.45 & 0.16 \\
\hline & $J_{\mathrm{HH}}$ & 0.01 & -0.30 & 0.30 & 0.01 \\
\hline \multirow{3}{*}{4} & $J_{\mathrm{CH}}{ }^{(a)}$ & 0.03 & -0.55 & 0.68 & 0.15 \\
\hline & $J_{\mathrm{CH}}{ }^{(\mathrm{b})}$ & 0.06 & -0.49 & 0.60 & 0.17 \\
\hline & $J_{\mathrm{HH}}$ & 0.01 & -0.57 & 0.73 & 0.17 \\
\hline \multirow{5}{*}{5} & $J_{\mathrm{CH}}{ }^{(\mathrm{a})}$ & 0.08 & -0.46 & 0.52 & 0.15 \\
\hline & $J_{\mathrm{CH}}{ }^{(\mathrm{d})}$ & 0.11 & -0.33 & 0.44 & 0.21 \\
\hline & $J_{\mathrm{CH}}{ }^{(\mathrm{e})}$ & 0.09 & -0.31 & 0.41 & 0.19 \\
\hline & $J_{\mathrm{CH}}{ }^{(f)}$ & 0.10 & -0.30 & 0.40 & 0.20 \\
\hline & $J_{\mathrm{HH}}$ & 0.00 & -0.30 & 0.30 & 0.00 \\
\hline \multirow{3}{*}{6} & $J_{\mathrm{CH}}{ }^{(a)}$ & 0.07 & -0.36 & 0.46 & 0.17 \\
\hline & $J_{\mathrm{CH}}{ }^{(\mathrm{b})}$ & 0.09 & -0.36 & 0.44 & 0.17 \\
\hline & $J_{\mathrm{HH}}$ & 0.00 & -0.30 & 0.30 & 0.00 \\
\hline
\end{tabular}

${ }^{\mathrm{a}}$ Substituted Carbons. ${ }^{\mathrm{b}}$ Unsubstituted carbons. ${ }^{\mathrm{c}}$ Ortho carbons. ${ }^{\mathrm{d}}$ Meta carbons. ${ }^{\mathrm{e}}$ Para carbon. 
First of all let us consider the unsubstituted dimer 1. In this case, as already found in our previous work [1,2], the through-space $J_{\mathrm{CH}}$ spin-spin coupling is of the order of $0.2 \mathrm{~Hz}$ and stems equally from a non negligible FC term $(0.09 \mathrm{~Hz})$ and from a non exact cancellation of the spinorbit contributions $(0.10 \mathrm{~Hz})$. The $J_{\mathrm{HH}}$ spin-spin coupling, instead, is essentially zero, because the FC term is zero, and because of an exact compensation of the spin-orbit contributions.

The substitution of the hydrogen on the $\mathrm{CH}$ bond with fluorine atoms leads to dimer $\mathbf{2}$. Here, we observe a slight increase in the total $J_{\mathrm{CH}}$, up to $0.21 \mathrm{~Hz}$ and a large increase of the $J_{\mathrm{HH}}$ spinspin coupling to $0.16 \mathrm{~Hz}$. However, the increase is entirely due to the spin-orbit contribution; in fact the FC term of the $J_{\mathrm{CH}}$ coupling is strongly decreased by $50 \%$, while the FC term of $J_{\mathrm{HH}}$ remains zero. For dimer $\mathbf{3}$, the results differ according to the substitution on the carbon atoms of the donor aromatic moiety. For both types of carbons, a decrease of $J_{\mathrm{CH}}$ is observed compared to model dimer 1. This decrease is due to (i) a decrease in the value of the FC term for the substituted carbons and (ii) to a decrease in the spin orbit contribution for the unsubstituted carbons. Once again, the $J_{\mathrm{HH}}$ values are unaltered, the FC term remains zero, and the spin-orbit contributions exactly cancel each other.

The results of model dimer $\mathbf{4}$ show the same trend compared with $\mathbf{3}$, as observed when going from dimer 1 to dimer 2 . In fact, there is a slight increase in the total through-space spin-spin coupling, which is due entirely to the spin-orbit contribution. The FC term is reduced by about $50 \%$ compared to dimer 3 , but an increase in the spin-orbit contributions compensate for this reduction, particularly for the $J_{\mathrm{HH}}$ coupling, which rises to a value of $0.17 \mathrm{~Hz}$.

In dimer 5, the benzene ring bears an electron-withdrawing substituent, $-\mathrm{NO}_{2}$. The effect of this substitution appears to be almost irrelevant. Compared to the unsubstituted dimer $\mathbf{1}$, the presence of the nitro group slightly alters the various contributions to the total through-space coupling, but no major changes in either $J_{\mathrm{CH}}$ or in $J_{\mathrm{HH}}$ can be detected.

Finally, the mesitylene-methane dimer $\mathbf{6}$ does not show any relevant difference compared to the unsubstituted model dimer $\mathbf{1}$.

\section{Discussion}

Apparently, none of the attempted substitutions, either in the donor $\pi$ system or in the acceptor $\mathrm{CH}$ bond, was capable of producing an increase in the strength of the through-space spin-spin coupling. Even more surprisingly, the FC contribution appeared to be unaltered by substituents or, more often, strongly diminished. This result may appear to be counter-intuitive, since the Fermi contact term is proportional to the amount of delocalization of the electrons between the nuclei involved in the coupling. In this respect, $\mathbf{3}$ certainly displays larger charge transfer character, from the 1,3,5-trihydroxybenzene HOMO to the trifluoromethane LUMO, than does (unsubstituted) 1. However, we should recall that the FC contribution is transmitted by $\sigma$-type orbitals, since it requires the probability, and therefore the wave function of the electron, to be non-zero at the nucleus. On the other hand, the mesomeric electronic effects on the benzene ring 
only involve the aromatic $\pi$ system, which has a nodal plane on the carbon atoms. These effects, therefore, should not be invoked to explain the results.

If we concentrate on $\sigma$-type orbitals it is not surprising that substitution with more electronegative atoms produce a reduction of the Fermi contact contribution. The more electronegative atom (e.g., oxygen in 3) attracts the $s$ electron density of the carbon, thereby reducing the already small overlap with the $s$ electron density of the hydrogen of the $\mathrm{CH}$ bond. Therefore, the coupling with the substituted carbon atom of 3 decreases to $0.04 \mathrm{~Hz}$. Substitution of hydrogens by fluorine on the $\mathrm{CH}$ bond has exactly the same effect, thereby polarizing the $s$ electron density far away from the internuclear region, thus decreasing even more the FC term associated with all carbon atoms of 1,3,5-trihydroxybenzene in $\mathbf{4}$.

Despite the opposite mesomeric effect, already mentioned, of the nitro group with respect to the hydroxy group, nitro substitution in $\mathbf{5}$ produces the same effect as the other substitutions. Thus, the nitro group polarizes the $s$ electron density towards the periphery of the dimer and reduces the overlap which is responsible for the small through-space FC contribution to the coupling.

Since all substitutions with a more electronegative atom on the aromatic ring and on the $\mathrm{CH}$ bond lead to a decrease of the FC term, and often a decrease of the overall coupling, we also investigated the effect of alkyl substitution, as in 6. However, this produced no significant changes in the through-space coupling. The inductive effect, which may be expected to be effective in this case, seems not to be large enough to induce an increase in the coupling.

At this juncture, it is instructive to visualize the "small" overlap between the $s$ orbitals of the hydrogen of the $\mathrm{CH}$ bond and the adjacent carbon atom in the aromatic ring. In Figure 2 we show a molecular orbital obtained as a linear combination of the $1 \mathrm{~s}, 2 \mathrm{~s}$ and $3 \mathrm{~s}$ basis function of the carbon atoms of the aromatic ring and the $1 \mathrm{~s}$ and $2 \mathrm{~s}$ orbitals of the hydrogen of the $\mathrm{CH}$ bond, for the dimer 2 . 


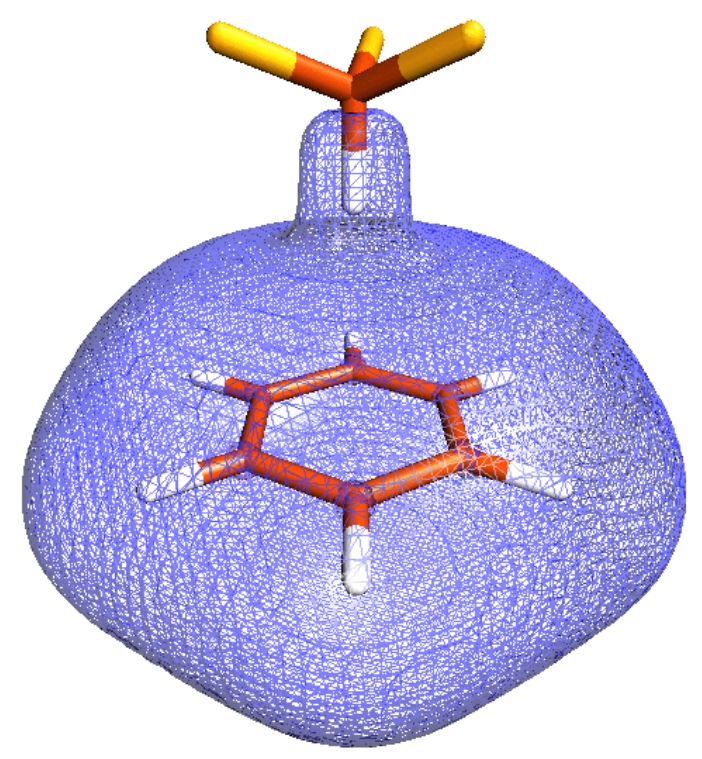

Figure 2. Molecular orbital of model dimer 2. Contour level is 0.002; HF/6-31G* level of theory.

The contour value in Figure 2 is set to 0.002, which indicates the small overlap that exists between the two $s$ electron densities, one from the donor and one from the acceptor. However, when present, this small overlap is responsible for the observed Fermi contact contribution.

The trends observed in the spin-orbit contribution are more difficult to rationalize. It is interesting to notice that the substitution of the $\mathrm{CH}$ bond with fluorine produces an increase in the otherwise negligible $J_{\mathrm{HH}}$ coupling.

\section{Conclusions}

We have investigated substituent effects on the through-space spin-spin coupling on the donor and acceptor moieties of a series of $\mathrm{CH} / \pi$ interacting dimers. The results of DFT calculations can be interpreted qualitatively, limited to the Fermi contact contribution, by considering the difference in electronegativity of the substituent ligands rather then their electronic mesomeric effects. Therefore, we do not observe any difference between "activating" and "deactivating" substituents with respect to the benzene ring. In contrast, because in all cases the substituent ligand is more electronegative than hydrogen, the effect of the substituent is to reduce the Fermi contact contribution to the coupling. This reduction may be offset by an increase in spin-orbit contribution. However, no significant increase in the overall coupling can be induced by the kind of substitution we have considered. These results suggest that a larger value of the coupling, in particular an increase in the Fermi contact contribution, could be present in systems where the 
hydrogen and carbon atoms are bonded to an electropositive element, as, e.g., in organometallic compounds.

\section{References}

1. Bagno, A.; G. Saielli and G.Scorrano, Angew. Chem. Int. Ed. Eng. 2001, 40, 2532.

2. Bagno, A.; Saielli, G.; Scorrano, G. Chem.Eur. J. 2002 in press.

3. Liu, A.; Majumdar, A.; Jiang, F.; Chernichenko, N.; Skripkin, E.; Patel, D. J. J. Am. Chem. Soc. 2000, 122, 9289.

4. Gaussian 98 (Revision A.7), Frisch, M. J.; Trucks, G. W.; Schlegel, H. B.; Scuseria, G. E.; Robb, M. A.; Cheeseman, J. R.; Zakrzewski, V. G.; Montgomery, J. A.; Stratmann, R. E.; Burant, J. C.; Dapprich, S.; Millam, J. M.; Daniels, A. D.; Kudin, K. N.; Strain, M. C.; Farkas, O.; Tomasi, J.; Barone, V.; Cossi, M.; Cammi, R.; Mennucci, B.; Pomelli, C.; Adamo, C.; Clifford, S.; Ochterski, J.; Petersson, G. A.; Ayala, P. Y.; Cui, Q.; Morokuma, K.; Malick, D. K.; Rabuck, A. D.; Raghavachari, K.; Foresman, J. B.; Cioslowski, J.; Ortiz, J. V.; Stefanov, B. B.; Liu, G.; Liashenko, A.; Piskorz, P.; Komaromi, I.; Gomperts, R.; Martin, R. L.; Fox, D. J.; Keith, T.; Al-Laham, M. A.; Peng, C. Y.; Nanayakkara, A.; Gonzalez, C.; Challacombe, M.; Gill, P. M. W.; Johnson, B. G.; Chen, W.; Wong, M. W.; Andres, J. L.; Head-Gordon, M.; Replogle, E. S.; Pople, J. A. Gaussian, Inc., Pittsburgh, PA, 1998.

5. Nishio, M.; Hirota, M.; Umezawa, Y. The $\mathrm{CH} / \pi$ Interaction, Evidence, Nature and Consequences, Wiley-VCH: New York, NY, 1998.

6. (a) Salahub, D. R.; Fournier, R.; Mlynarski, P.; Papai, I.; St-Amant, A. ; Ushio, J. In Density Functional Methods in Chemistry; Labanowski, J.; Andzelm, J., Eds; Springer: New York, 1991. (b) St-Amant, A.; Salahub, D. R. Chem. Phys. Lett. 1990, 169, 387. (c) Malkin, V. G.; Malkina, O. L.; Casida, M. E.; Salahub, D. R. J. Am. Chem. Soc. 1994, 116, 5898. (d) Malkin, V. G.; Malkina, O. L.; Eriksson, L. A.; Salahub, D. R. In Modern Density Functional Theory: A Tool For Chemistry; Seminario, J. M.; Politzer, P., Eds; Elsevier: Amsterdam, 1995; Vol. 2. (e) Malkin, V. G.; Malkina, O. L.; Salahub, D. R. Chem. Phys. Lett. 1994, 221, 91. (f) Malkina, O. L.; Salahub, D. R.; Malkin, V. G. J. Chem. Phys. 1996, 105, 8793. 